\title{
Dynamic contrast enhanced T1 MRI perfusion differentiates pseudoprogression from recurrent glioblastoma
}

\author{
Alissa A. Thomas ${ }^{1}$, Julio Arevalo-Perez ${ }^{2}$, Thomas Kaley ${ }^{1,5}$, John Lyo ${ }^{2,5}$, Kyung K. Peck ${ }^{3}$, \\ Weiji Shi ${ }^{4}$, Zhigang Zhang ${ }^{4}$, and Robert J. Young ${ }^{2,5}$ \\ Robert J. Young: youngr@mskcc.org \\ ${ }^{1}$ Department of Neurology, Memorial Sloan Kettering Cancer Center, New York, NY 10065, USA \\ 2Department of Radiology, Memorial Sloan Kettering Cancer Center, New York, NY 10065, USA \\ ${ }^{3}$ Department of Medical Physics, Memorial Sloan Kettering Cancer Center, New York, NY 10065, \\ USA
}

${ }^{4}$ Department of Epidemiology \& Biostatistics, Memorial Sloan Kettering Cancer Center, New York, NY 10065, USA

${ }^{5}$ The Brain Tumor Center, Memorial Sloan Kettering Cancer Center, New York, NY 10065, USA

\begin{abstract}
Pseudoprogression may present as transient new or increasing enhancing lesions that mimic recurrent tumors in treated glioblastoma. The purpose of this study was to examine the utility of dynamic contrast enhanced T1 magnetic resonance imaging (DCE MRI) in differentiating between pseudoprogression and tumor progression and devise a cut-off value sensitive for pseudoprogression. We retrospectively examined 37 patients with glioblastoma treated with radiation and temozolomide after surgical resection that then developed new or increasing enhancing lesion(s) indeterminate for pseudoprogression versus progression. Volumetric plasma volume ( $\mathrm{Vp}$ ) and time-dependent leakage constant (Ktrans) maps were measured for the enhancing lesion and the mean and ninetieth percentile histogram values recorded. Lesion outcome was determined by clinical follow up with pseudoprogression defined as stable disease not requiring new treatment. Statistical analysis was performed with Wilcoxon rank-sum tests. Patients with pseudoprogression $(\mathrm{n}=13)$ had $\mathrm{Vp}($ mean $)=2.4$ and $\mathrm{Vp}(90 \%$ tile $)=3.2$; and Ktrans $($ mean $)=3.5$ and Ktrans $(90 \%$ tile $)=4.2$. Patients with tumor progression $(\mathrm{n}=24)$ had $\mathrm{Vp}$ $($ mean $)=5.3$ and $\mathrm{Vp}(90 \%$ tile $)=6.6$; and Ktrans $($ mean $)=7.4$ and Ktrans $(90 \%$ tile $)=9.1$. Compared with tumor progression, pseudoprogression demonstrated lower Vp perfusion values ( $\mathrm{p}$ $=0.0002$ ) with a $\mathrm{Vp}$ (mean) cutoff $<3.7$ yielding $85 \%$ sensitivity and $79 \%$ specificity for
\end{abstract}

Correspondence to: Robert J. Young, youngremskcc.org.

Alissa A. Thomas and Julio Arevalo-Perez have contributed equally.

Electronic supplementary material The online version of this article (doi:10.1007/s11060-015-1893-z) contains supplementary material, which is available to authorized users.

Author contributions

Drs. Thomas, Arevalo-Perez, Kaley, and Young participated in the design the study. Drs. Thomas, Arevalo-Perez, Peck, Shi, Zhang, and Young participated in the study conduct and gathered and interpreted the data. All authors were involved in the preparation of the report, and reviewed both the draft and final versions.

Conflict of interest None. 
pseudoprogression. Ktrans (mean) of $>3.6$ had a $69 \%$ sensitivity and $79 \%$ specificity for disease progression. DCE MRI shows lower plasma volume and time dependent leakage constant values in pseudoprogression than in tumor progression. A cut-off value with high sensitivity for pseudoprogression can be applied to aid in interpretation of DCE MRI.

\section{Keywords}

Glioblastoma; DCE MRI; Pseudoprogression; Perfusion

\section{Introduction}

Accurate differentiation between pseudoprogression (PsP) and recurrent tumor is essential for optimal treatment management in glioblastoma. The new and/or increased enhancing lesions of PsP after external beam radiation therapy (RT), particularly when combined with temozolomide (TMZ) chemotherapy [1, 2], mimic true tumor progression (PD). With RT plus concomitant and adjuvant TMZ [3], PsP occurs in an estimated 20-30\% of all patients treated for malignant glioma [4]. The risk for PsP is higher in patients with O6methylguanine-methyltransferase (MGMT) promoter methylation [5], which is known to increase the tumor's sensitivity to the alkylating effects of TMZ [6] and is associated with increased risk of radiation-induced side effects.

PsP generally appears on MRI within the first 3 months of completing radiation therapy, but can occur 6 months after radiation therapy or longer $[2,7]$. Confirmation of PsP requires either stability or improvement in enhancement on follow up MRI in the absence of a change in treatment, or pathologic demonstration of treatment related necrosis on surgical resection. Progression-free survival (PFS) for newly diagnosed glioblastoma is approximately 6-7 months [3]. After accounting for a median of 5 weeks from diagnosis to start of radiation and a standard 6 week course of radiation, the time frame for PsP overlaps significantly with when glioblastoma is most likely to progress. A non-invasive means of distinguishing PD from PsP would aid in making prompt informed treatment decisions by avoiding the delay necessary for follow up scans, and could spare some patients the morbidity related to repeat surgery.

PsP is likely a reflection of increased inflammation and disruption of the blood brain barrier (BBB) caused by radiation and enhanced by concurrent use of temozolomide [4]. Due to the disruption of the BBB, both PD and PsP can present with increased gadolinium enhancement on MRI. Perfusion is the process of delivering blood, oxygen, and nutrients to tissue via the blood flow. Because glioblastoma is a highly vascular tumor characterized by endovascular proliferation and angiogenesis, we would expect that progressive glioblastoma would have increased perfusion relative to PsP. Dynamic MRI can be used to measure tissue perfusion through fast repeated acquisitions of the same tissue volume before administration of gadolinium an during its passage through the tissue [8]. Two types of sequences can be used: T2* weighted echo planar imaging (dynamic susceptibility contrast or DSC) or T1weighted 2D/three dimensional fast gradient echo sequence (dynamic contrast enhanced or DCE) [8]. DCE has the advances of less sensitivity to susceptibility artifacts, better 
quantification of BBB permeability, and more quantitative results $[9,10]$. DCE perfusion imaging is becoming more widely available, but standardized metrics for interpreting imaging across institutions is lacking. The purpose of this study was to examine the utility of T1-weighted DCE-MRI in differentiating between PsP and PD. Our goal was to devise a cut-off value for the perfusion parameters that would be sensitive for PsP. We hypothesized that PsP would correlate with lower perfusion parameters as measured by Ktrans and Vp.

\section{Materials and methods}

\section{Study design}

This was an IRB approved retrospective single institution study.

\section{Patient selection}

As summarized in Fig. 1, we reviewed all patients treated from glioblastoma between March 2011 and October 2013. We included patients with the following characteristics: (1) newly diagnosed primary glioblastoma by histopathology; (2) had undergone gross total or subtotal resection or biopsy of their tumor; (3) had received RT with concomitant and adjuvant TMZ chemotherapy; (4) developed enlarging and/or new enhancing lesion(s) inside the radiation therapy (RT) field; and (5) had pre-operative and follow up DCE perfusion scans available for analysis. All patients received at least three cycles of adjuvant TMZ. Steroid use was allowed in accordance with standard of care practices. The charts were reviewed to determine the treatment course including date of diagnosis, surgical resection, RT, chemotherapy, dates of disease progression, and date of death. Extent of resection was determined from the post-operative MRI performed within $72 \mathrm{~h}$ of surgery. If no MRI was available, extent of resection was determine from the operative report.

\section{MRI protocol}

Patients were required to have MRI with DCE performed on 1.5 and 3.0 -T magnets (Signa HDx, Signa Excite, Discovery 750, GE Healthcare, Milwaukee, WI, USA) using a standard 8 channel head coil. A bolus of gadopentetate dimeglumine (Magnevist, Bayer Healthcare Pharmaceuticals, Whippany, NJ, USA) was administered by a power injector at 0.1 $\mathrm{mmol} / \mathrm{kg}$ body weight and at a rate of $2-3 \mathrm{~mL} / \mathrm{s}$ followed by a $40 \mathrm{~mL}$ saline bolus. DCE MRI of the brain was acquired with a 3D T1-weighted spoiled gradient recalled sequence (TR 4-5 ms; TE 1-2 ms; slice thickness $5 \mathrm{~mm}$; FA 25; FOV $24 \mathrm{~cm}$; matrix $128 \times 128$; temporal resolution $(\Delta \mathrm{t}) 5-6 \mathrm{~s}$. Ten phases were acquired for pre-injection time delay and 30 phases for post-injection were obtained with an acquisition time of $5 \mathrm{~min}$. Matching T1weighted $(\mathrm{TR} / \mathrm{TE}=600 / 8 \mathrm{~ms}$; thickness $=5 \mathrm{~mm})$ and T2-weighted $(\mathrm{TR} / \mathrm{TE}=4000 / 102 \mathrm{~ms}$; thickness $=5 \mathrm{~mm}$ ) spin-echo images were obtained.

\section{Image analysis}

DCE MRI data and matching contrast T1-weighted images were transferred to an off-line workstation and processed using available commercial software (Nordic Ice; Nordic NeuroLab, Bergen, Norway). A trained operator performed all DCE MRI analyses while blinded to the patients' clinical status, MGMT status and lesion diagnosis. Data preprocessing consisted of noise correction, motion artifact rectification and semi-automated 
arterial input function (AIF) selection. These steps permitted to optimize the signal-to-noise ratio and the AIF by choosing a suitable artery to typify the input function curve and the concentration-time curve [11]. The two-compartment model proposed by Tofts was used for pharmacokinetic parameter calculation of time-dependent leakage (Ktrans) and blood plasma volume (Vp) [12]. Results were displayed as parametric maps. 2D regions-of-interest (ROI) were drawn around the enhancing lesion on each contrast T1-weighted slice to generate a volume-of-interest (VOI) for the entire enhancing lesion. Meticulous care was taken not to include large vessels or hemorrhage in the region. The VOIs were transferred from the contrast images onto the Ktrans and Vp maps, and the resulting measurements binned into histograms. The histograms were then normalized to the contralateral normal brain parenchyma. Based on clinical experience, the mean and $90 \%$ percentile histogram values for Ktrans and $\mathrm{Vp}$ were recorded.

\section{Lesion diagnosis}

Dates of PD and PsP were determined based on review of clinic and hospital admission notes and radiology reports. The patients in this sample did not undergo re-resection. In the absence of pathology, the clinical diagnosis of PD or PsP was made after complete chart and imaging review. The diagnosis of PsP was made if the follow up MRI brain was stable or improved and no change in treatment was required for a minimum of two additional adjuvant cycles of TMZ [5]. The diagnosis of PD was made if imaging or clinical worsening prompted a change in treatment.

\section{Statistical analysis}

Univariate analysis using Wilcoxon rank-sum test was performed to determine the relative utility of perfusion imaging in predicting PsP versus PD. The cutoffs of the perfusion imaging parameters were selected using the receiver operating characteristic (ROC) method. The area under the ROC curve (AUC) of the perfusion parameters were compared using the Delong's test. Univariate analyses were also used to determine the relationship between PsP and MGMT status, and PsP and overall survival (OS). OS among the groups was calculated from the date of surgery to the date of death or last follow up, and was estimated using the Kaplan-Meier method. OS was compared between the PsP and PD group using the Cox proportional hazards model ra and adjusted for age, Karnofsky performance score (KPS), and MGMT methylation status; PsP status was treated as a time dependent variable in this analysis. The statistical analysis was performed with the software SAS version 9.2 (SAS Institute Cary, NC, USA) and r packages ROCR and pROC (version 3.1.1). Significance level was set to $p$ value $=0.05$.

\section{Results}

\section{Patient characteristics}

We identified thirty-seven patients meeting all inclusion criteria; their demographics are summarized in Table 1. Two patients (5\%) had gross total resections. Extent of resection did not correlate with OS ( $\mathrm{p}=0.50)$. Information on MGMT promoter methylation status was available for 36 of the 37 patients. MGMT promoter methylation was detected in 15 patients (41.7\%), 7 of whom were in the PsP group ( $47 \%$ of the methylated patients, and 58 
$\%$ of patients with PsP), and 8 in the PD group ( $33 \%$ ). The trend toward more MGMT methylated patients in the PsP group was not statistically significant $(\mathrm{p}=0.15)$. PsP was observed in the first 12 weeks after completion of RT in 11 of 13 (84.6\%) patients and within 24 weeks in all patients. All patients eventually developed PD. Examples of PsP and PD can be seen in Fig. 2 and Supplemental Fig. 1.

\section{DCE}

As summarized in Tables 2 and 3, the median normalized blood plasma volume ( $\mathrm{Vp}(\mathrm{mean})$ ) was lower for PsP at 2.4 than for PD at 5.3 with $\mathrm{p}=0.0002$. The median normalized $\mathrm{Vp}$ (90 \%) was also lower for PsP at 3.2 than for PD at 6.6 with $\mathrm{p}<0.0001$, as was the Ktrans for $\mathrm{PsP}$ at 3.5 compared to $\mathrm{PD}$ at 7.4 with $\mathrm{p}=0.002$. To optimize specificity and sensitivity, a $\mathrm{Vp}$ (mean) threshold of $>3.7$ indicated PD had $85 \%$ sensitivity and $79 \%$ specificity for PsP, a Vp (90\%) threshold $>3.9$ had $92 \%$ specificity and $85 \%$ sensitivity, and Ktrans(mean) $>3.6$ had $79 \%$ specificity and $69 \%$ sensitivity (Fig. 3). There was a trend that AUC Vp (90 $\%$ ) was relatively greater than AUC Ktrans (mean) (0.904 vs. 0.808, p = 0.07).

We also compared the change in perfusion parameters for the indeterminate lesion (PsP vs. $\mathrm{PD}$ ) from the baseline pre-operative tumor (PsP versus PD) (Tables 2, 3). The median decrease between scans was greater for $\mathrm{Vp}(90 \%) \%$ in PsP at $-39.6 \%$ (range, -70.3 to +167.1 ) than in PD at $-2.6 \%$ (range, -59.9 to +175.2 ) with $\mathrm{p}=0.02$. Changes in the other Vp (mean), Ktrans (mean) and Ktrans $(90 \%)$ were not significant with $\mathrm{p}>0.19$.

\section{Overall survival}

Seventeen patients died and 20 were alive at last follow-up. The median follow-up time for survivors was 15.7 months (range, 8.5-34.8). On univariate analysis, PsP had superior OS compared to $\mathrm{PD}(\mathrm{HR}=0.22,95 \% \mathrm{CI} 0.06-0.78 ; \mathrm{p}=0.02$ by Cox proportional hazards model). There was no significant difference in OS between PsP and PD (HR 0.44, 95 \% CI $0.10-1.82, \mathrm{p}=0.25)$, however, after adjusting for age, KPS, and MGMT status.

\section{Discussion}

We retrospectively examined the utility of DCE MRI to non-invasively distinguish between PsP and PD in patients with treated glioblastoma. We found that the patients with PsP had lower volumetric histogram measures of $\mathrm{Vp}$ and Ktrans than did patients with PD. This suggests that DCE MRI has an important role in the management of glioblastoma patients with indeterminate lesions that worsen on conventional imaging after RT.

The enhancing lesions of PsP are caused by radiation-induced endothelial injury, vascular dilatation and fibrinoid necrosis with inflammatory blood-brain barrier disruption [13]. In contrast, the enhancing lesions of PD are due to actively growing tumor with neoangiogenesis, vascular proliferation and neoplastic blood-brain barrier disruption. Accurate differentiation between PsP and PD is critical for making informed treatment decisions. Since PsP spontaneously stabilizes or resolves, and suggests favorable accelerated tumor cell killing and improved outcome partially due to its association with methylated MGMT promoter status [5], patients with PsP should continue their current effective 
therapy. PD indicates that repeat surgery and/or new chemotherapy should be substituted for current ineffective therapy.

Other MRI techniques have been used to try to distinguish between PsP and PD. After examining nine signs based on conventional MRI images, only subependymal enhancement was found to have high specificity (93.3\%) for PsP although the low sensitivity (38.1\%) and low negative predictive value $41.8 \%$ limit its application in clinical practice [14]. DSC MRI with ferumoxytol has been used to define PsP [15], with relative cerebral blood volume (rCBV) $\leq .75$ predictive for PsP (sensitivity and specificity not reported). In another study using histogram analysis of both normalized cerebral blood volume (nCBV) and apparent diffusion coefficient (ADC), the 5th percentile values of ADC were able to distinguish between PD and PsP ( $p=0.044,0.011)$ but $n C B V$ was not [16].

DCE MRI has distinct advantages over DSC MRI that include greater spatial resolution, less artifact from sources of susceptibility (e.g., hemorrhage, bone, metal, air) and better estimations of vascular leakiness [17]. Using histogram analysis, we measured volumetric DCE MRI parameters through the entirety of the enhancing lesion, thereby presenting a more accurate depiction of the enhancing lesions while capturing some of the innate heterogeneity of these lesions usually lost with the use of large 2D ROIs. Use of the histogram technique also helps to filter out extraneous signal caused by areas of cystic and/or necrotic change in the tumor. In addition, the 3D volumetric approach mitigates the subjectivity of single 2D ROI based analysis. These attributes should enable better characterization of indeterminate lesions.

We found that lower mean and 90th percentile values for both Vp and Ktrans correlated with PsP. Our findings of increased Ktrans in disease progression are consistent with a recent study [18]. Yun et al. performed DCE MRI in 33 patient with glioblastoma and found lower mean Ktrans in PsP $\left(0.44 \mathrm{~min}^{-1}\right)$ than in PD $\left(0.23 \mathrm{~min}^{-1}, \mathrm{p}=0.004\right)$ [18]. After multivariate analysis, only mean Ktrans $<0.347 \mathrm{~min}^{-1}$ was able to predict PsP with $94 \%$ specificity and $59 \%$ sensitivity. While the authors also used a volumetric approach, the lack of significance for histogram derived percentile metrics, and for VP, may have been due to their use of absolute values rather than normalized ratios that help mitigate inter-scanner and interpatient variability. Similarly, in a study using DSC MRI to distinguish PD from PsP in high grade glioma, changes in relative cerebral blood volume ( $\mathrm{rCBV}$ ) over time were predictive, with PsP demonstrating an overall negative linear trend in $\mathrm{rCBV}$ and PD demonstrating a positive slope [19].

We also compared DCE MRI metrics of the indeterminate enhancing lesion to the baseline untreated lesion, but did not find any change predictive for PsP. This suggests that DCE MRI at a single time point, when the indeterminate enhancing lesion presents, is sufficient for determination of lesion etiology. While noncontributory for PsP, we recommend routine preoperative DCE MRI in glioma patients as it has been shown to be helpful in predicting glioma grade and prognosis [20], and to help target tissue sampling to the highest grade tissue within the tumor for histological evaluation. 
In this study, we elected to use a broad definition of PsP to allow for clinical generalizability. In their article in the Lancet, Brandsma and colleagues defined PsP as "subacute treatment-related reactions with or without clinical deterioration showing edema and sometimes contrast enhancement on MRI suggestive of tumor progression. Despite the clinical or radiological suggestion of tumor progression, these patients recovered or stabilized spontaneously, and often without permanent new deficits [2]." Some MRI studies have restricted this definition to the first post-radiation MRI scan or to changes on MRI seen within the first 12 weeks after completion of RT [14, 21-23], and this 12 week time frame for PsP has been used by the response assessment in neuro-oncology (RANO) working group. After re-examining the RANO criteria, however, a group recently found that nearly $30 \%$ of patients with PsP had MRI changes $>3$ months after finishing RT [15]. The authors suggested that PsP should be considered as a possible cause for radiologic changes regardless of the duration of time since radiotherapy. In our study, $85 \%$ of our patients ( $n=$ 11) experienced PsP within 12 weeks of completing radiation, and $100 \%$ within 24 months. The two patients who presented with PsP more than 12 weeks after RT did so at 14 and 23 weeks, respectively. After being categorized as PsP, these two patients remained stable with no change in therapy for 8-9 months, suggesting that despite the late presentation, the change on MRI was, in fact, PsP.

We found a trend toward increased MGMT promoter methylation in the pseudoprogressors. PsP was associated with increased survival only on univariate analysis, and this effect was lost when corrected for age, KPS, and MGMT status. By design, our sample overestimates PD to increase the sensitivity for diagnosing PsP. Lesions that prompted a change in therapy to bevacizumab because of uncertainly in diagnosis or clinical symptoms were all categorized as PD which may have skewed our correlations between MGMT hypermethylation and PsP.

A major limitation of our study is that our patients did not undergo re-resection at the time of the MRI indeterminate lesion; thus, our definition of PsP was clinical and imaging based and not pathologically proven. At times, the clinical documentation indicated that the MRI lesion was truly indeterminate, but rather than wait for follow up imaging, the clinician elected to change treatment from TMZ to bevacizumab. We classified all of these cases associated with change in therapy as progression. One of the limitations of this design is that by categorizing those patients who were immediately treated with bevacizumab as PD, we may have mislabeled some patients with severe and/or symptomatic PsP. Thus, one could conclude that low Vp and low Ktrans are associated with less severe PsP, but without tissue confirmation we cannot comment on the perfusion characteristics of severe PsP.

\section{Conclusion}

PsP remains a difficult clinical and imaging diagnosis. This study demonstrates that DCE MRI has a role in differentiating PsP from PD with utility for both Vp and Ktrans volumetric histogram metrics. We suggest that DCE MRI results should be incorporated into clinical treatment decisions especially when patients are unwilling or unable to undergo repeat surgery, or are hesitant to wait for follow up scans that might delay an appropriate change in treatment. As DCE MRI becomes widely available, simple cutoff values can help 
standardize interpretation of these scans and obviate the need for special tools and programs that may only be available in academic centers.

\section{Supplementary Material}

Refer to Web version on PubMed Central for supplementary material.

\section{Acknowledgments}

Julio Arevalo-Perez was supported by a grant from the Spanish foundation "Fundación Alfonso Martín Escudero". Weiji Shi and Zhigang Zhang's research was partly supported by an NIH Core Grant P30 CA008748.

\section{References}

1. Rogers LR. Neurologic complications of radiation. Continuum. 2012; 18(2):343354.10.1212/01.CON.0000413662.35174.a8 [PubMed: 22810131]

2. Brandsma D, Stalpers L, Taal W, Sminia P, van den Bent MJ. Clinical features, mechanisms, and management of pseudoprogression in malignant gliomas. Lancet Oncol. 2008; 9(5):453461.10.1016/S1470-2045(08)70125-6 [PubMed: 18452856]

3. Stupp R, Mason WP, van den Bent MJ, Weller M, Fisher B, Taphoorn MJ, Belanger K, Brandes AA, Marosi C, Bogdahn U, Curschmann J, Janzer RC, Ludwin SK, Gorlia T, Allgeier A, Lacombe D, Cairncross JG, Eisenhauer E, Mirimanoff RO, European Organisation for R, Treatment of Cancer Brain T, Radiotherapy G, National Cancer Institute of Canada Clinical Trials G. Radiotherapy plus concomitant and adjuvant temozolomide for glioblastoma. N Engl J Med. 2005; 352(10):987-996.10.1056/NEJMoa043330 [PubMed: 15758009]

4. Wen PY, Macdonald DR, Reardon DA, Cloughesy TF, Sorensen AG, Galanis E, Degroot J, Wick W, Gilbert MR, Lassman AB, Tsien C, Mikkelsen T, Wong ET, Chamberlain MC, Stupp R, Lamborn KR, Vogelbaum MA, van den Bent MJ, Chang SM. Updated response assessment criteria for high-grade gliomas: response assessment in neuro-oncology working group. J Clin Oncol. 2010; 28(11):1963-1972.10.1200/JCO.2009.26.3541 [PubMed: 20231676]

5. Brandes AA, Franceschi E, Tosoni A, Blatt V, Pession A, Tallini G, Bertorelle R, Bartolini S, Calbucci F, Andreoli A, Frezza G, Leonardi M, Spagnolli F, Ermani M. MGMT promoter methylation status can predict the incidence and outcome of pseudoprogression after concomitant radiochemotherapy in newly diagnosed glioblastoma patients. J Clin Oncol. 2008; 26(13):21922197.10.1200/JCO.2007.14.8163 [PubMed: 18445844]

6. Hegi ME, Diserens AC, Gorlia T, Hamou MF, de Tribolet N, Weller M, Kros JM, Hainfellner JA, Mason W, Mariani L, Bromberg JE, Hau P, Mirimanoff RO, Cairncross JG, Janzer RC, Stupp R. MGMT gene silencing and benefit from temozolomide in glioblastoma. N Engl J Med. 2005; 352(10):997-1003.10.1056/NEJMoa043331 [PubMed: 15758010]

7. Tran DK, Jensen RL. Treatment-related brain tumor imaging changes: so-called "pseudoprogression" vs. tumor progression: review and future research opportunities. Surg Neurol Int. 2013; 4(Suppl 3):S129-S135.10.4103/2152-7806.110661 [PubMed: 23682339]

8. Viallon M, Cuvinciuc V, Delattre B, Merlini L, Barnaure-Nachbar I, Toso-Patel S, Becker M, Lovblad KO, Haller S. State-of-the-art MRI techniques in neuroradiology: principles, pitfalls, and clinical applications. Neuroradiology. 2015; 57(5):441-467.10.1007/s00234-015-1500-1 [PubMed: 25859832]

9. Yeo DM, Oh SN, Jung CK, Lee MA, Oh ST, Rha SE, Jung SE, Byun JY, Gall P, Son Y, Son Y. Correlation of dynamic contrast-enhanced MRI perfusion parameters with angiogenesis and biologic aggressiveness of rectal cancer: preliminary results. J Magn Reson Imaging: JMRI. 2013; 41:474-480.10.1002/jmri.24541 [PubMed: 24375840]

10. Chung WJ, Kim HS, Kim N, Choi CG, Kim SJ. Recurrent glioblastoma: optimum area under the curve method derived from dynamic contrast-enhanced T1-weighted perfusion MR imaging. Radiology. 2013; 269(2):561-568.10.1148/radiol.13130016 [PubMed: 23878286] 
11. Jung SC, Yeom JA, Kim JH, Ryoo I, Kim SC, Shin H, Lee AL, Yun TJ, Park CK, Sohn CH, Park $\mathrm{SH}$, Choi SH. Glioma: application of histogram analysis of pharmacokinetic parameters from T1weighted dynamic contrast-enhanced MR imaging to tumor grading. AJNR Am J Neuroradiol. 2014; 35(6):1103-1110.10.3174/ajnr.A3825 [PubMed: 24384119]

12. Tofts PS, Brix G, Buckley DL, Evelhoch JL, Henderson E, Knopp MV, Larsson HB, Lee TY, Mayr NA, Parker GJ, Port RE, Taylor J, Weisskoff RM. Estimating kinetic parameters from dynamic contrast-enhanced T(1)-weighted MRI of a diffusable tracer: standardized quantities and symbols. J Magn Reson Imaging: JMRI. 1999; 10(3):223-232. [PubMed: 10508281]

13. Hopewell JW, Calvo W, Jaenke R, Reinhold HS, Robbins ME, Whitehouse EM. Microvasculature and radiation damage. Recent Results Cancer Res. 1993; 130:1-16. [PubMed: 8362079]

14. Young RJ, Gupta A, Shah AD, Graber JJ, Zhang Z, Shi W, Holodny AI, Omuro AM. Potential utility of conventional MRI signs in diagnosing pseudoprogression in glioblastoma. Neurology. 2011; 76(22):1918-1924.10.1212/WNL.0b013e31821d74e7 [PubMed: 21624991]

15. Nasseri M, Gahramanov S, Netto JP, Fu R, Muldoon LL, Varallyay C, Hamilton BE, Neuwelt EA. Evaluation of pseudoprogression in patients with glioblastoma multiforme using dynamic magnetic resonance imaging with ferumoxytol calls RANO criteria into question. Neuro-oncology. 2014; 16(8):1146-1154.10.1093/neuonc/not328 [PubMed: 24523362]

16. Song YS, Choi SH, Park CK, Yi KS, Lee WJ, Yun TJ, Kim TM, Lee SH, Kim JH, Sohn CH, Park $\mathrm{SH}$, Kim IH, Jahng GH, Chang KH. True progression versus pseudoprogression in the treatment of glioblastomas: a comparison study of normalized cerebral blood volume and apparent diffusion coefficient by histogram analysis. Korean J Radiol. 2013; 14(4):662-672.10.3348/kjr. 2013.14.4.662 [PubMed: 23901325]

17. Sourbron S, Ingrisch M, Siefert A, Reiser M, Herrmann K. Quantification of cerebral blood flow, cerebral blood volume, and blood-brain-barrier leakage with DCE-MRI. Magn Reson Med. 2009; 62(1):205-217.10.1002/mrm.22005 [PubMed: 19449435]

18. Yun TJ, Park CK, Kim TM, Lee SH, Kim JH, Sohn CH, Park SH, Kim IH, Choi SH. Glioblastoma treated with concurrent radiation therapy and temozolomide chemotherapy: differentiation of true progression from pseudoprogression with quantitative dynamic contrast-enhanced MR imaging. Radiology. 2015; 274(3):830-840.10.1148/radiol.14132632 [PubMed: 25333475]

19. Boxerman JL, Ellingson BM, Jeyapalan S, Elinzano H, Harris RJ, Rogg JM, Pope WB, Safran H. Longitudinal DSC-MRI for distinguishing tumor recurrence from pseudoprogression in patients with a high-grade glioma. Am J Clin Oncol. 201410.1097/COC.0000000000000156

20. Arvinda HR, Kesavadas C, Sarma PS, Thomas B, Radhakrishnan VV, Gupta AK, Kapilamoorthy TR, Nair S. Glioma grading: sensitivity, specificity, positive and negative predictive values of diffusion and perfusion imaging. J Neurooncol. 2009; 94(1):87-96.10.1007/s11060-009-9807-6 [PubMed: 19229590]

21. Linhares P, Carvalho B, Figueiredo R, Reis RM, Vaz R. Early pseudoprogression following chemoradiotherapy in glioblastoma patients: the value of RANO evaluation. J Oncol. 2013; 2013:690585.10.1155/2013/690585 [PubMed: 24000284]

22. Taal W, Brandsma D, de Bruin HG, Bromberg JE, Swaak-Kragten AT, Smitt PA, van Es CA, van den Bent MJ. Incidence of early pseudo-progression in a cohort of malignant glioma patients treated with chemoirradiation with temozolomide. Cancer. 2008; 113(2):405-410.10.1002/cncr. 23562 [PubMed: 18484594]

23. Sanghera P, Perry J, Sahgal A, Symons S, Aviv R, Morrison M, Lam K, Davey P, Tsao MN. Pseudoprogression following chemoradiotherapy for glioblastoma multiforme. Can J Neurol Sci. 2010; 37(1):36-42. [PubMed: 20169771] 


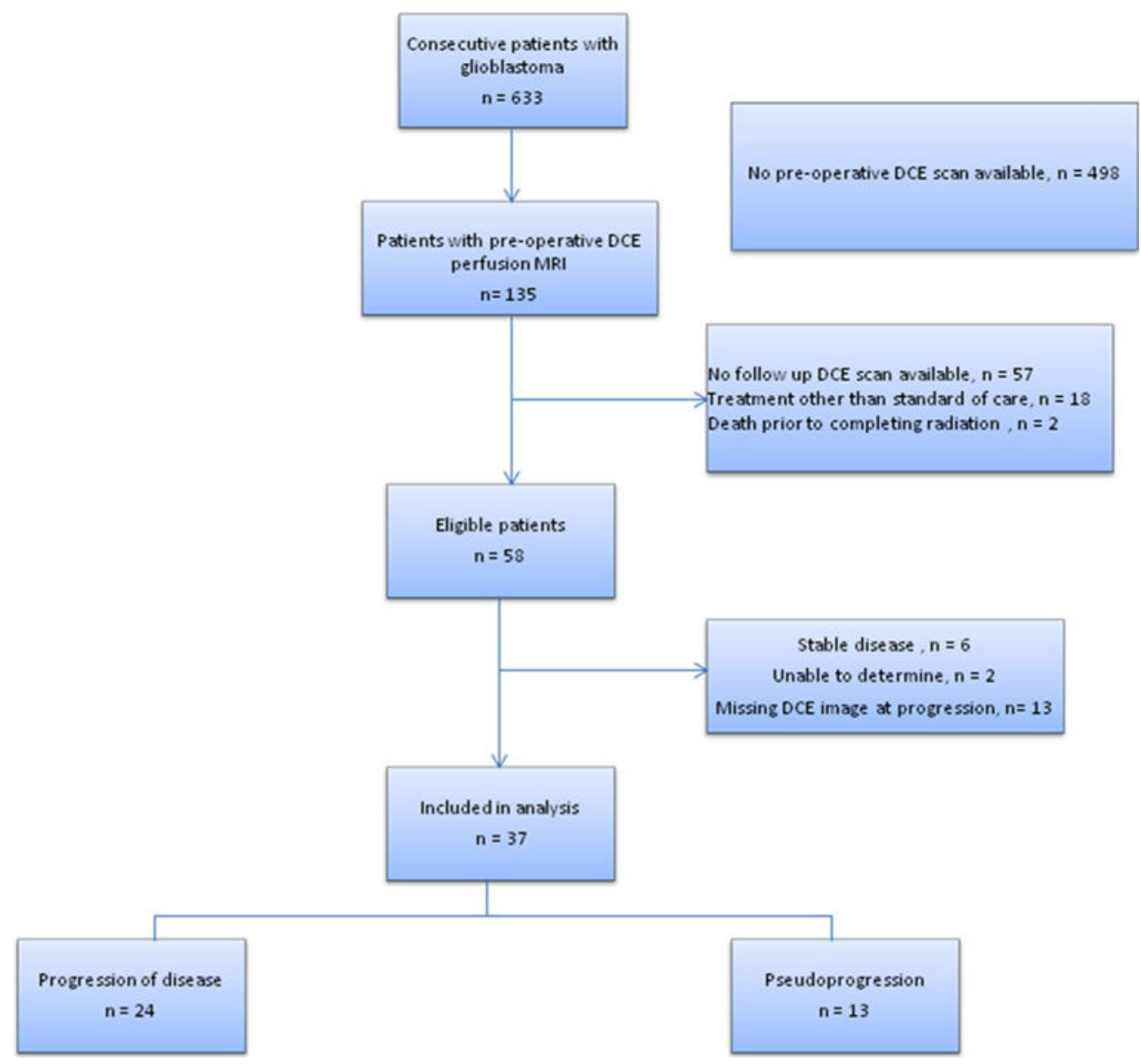

Fig. 1.

Patient cohort selection 


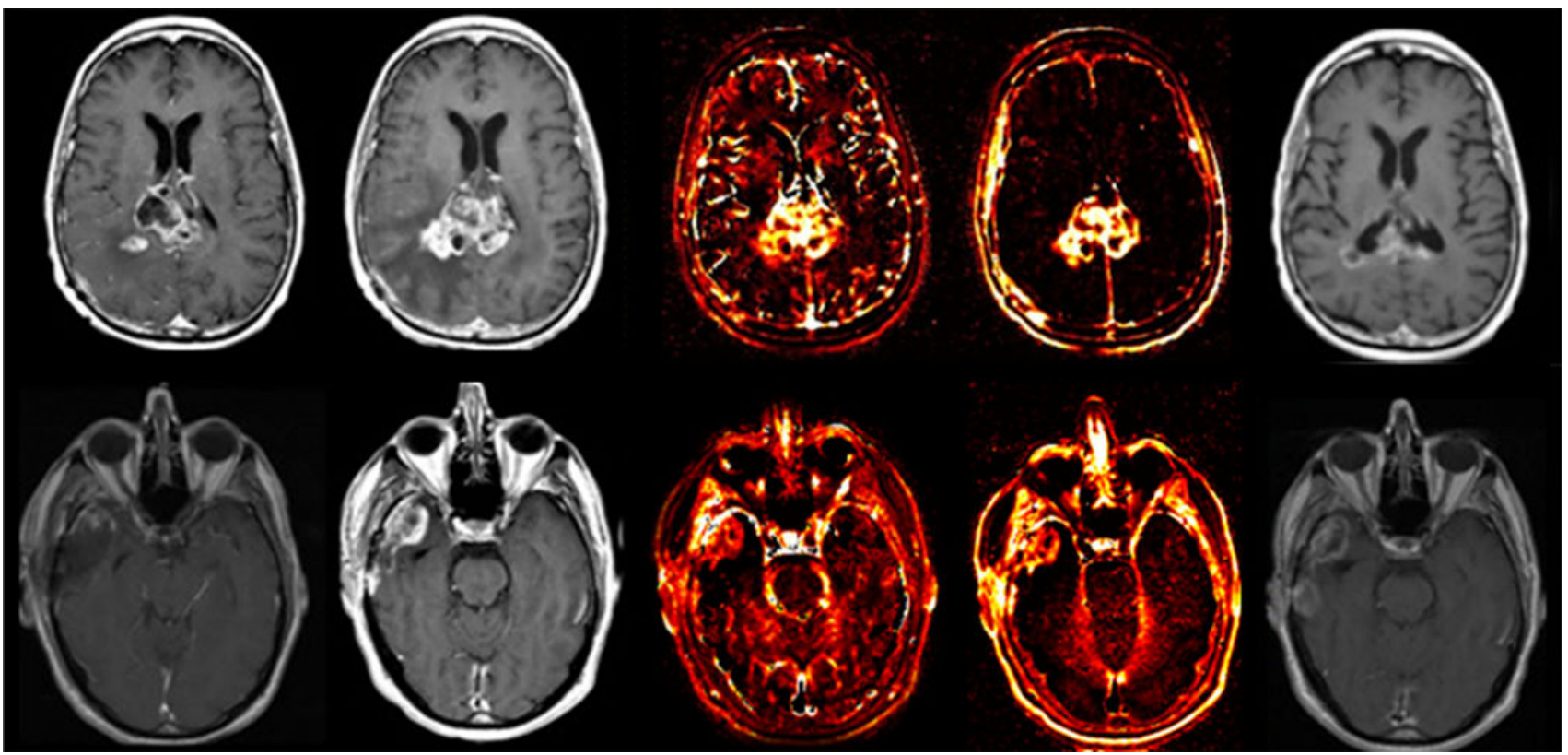

Fig. 2.

a (top) Example of perfusion imaging at disease progression. From left to right: a Axial T1weighted post-contrast image from the first post-radiation MRI; $b$ Axial T1-weighted postcontrast image 3 months after radiation therapy; $c$ The corresponding blood plasma volume $(\mathrm{Vp})$ parametric map, $\mathrm{Vp}$ mean is 13.8 ; $d$ Corresponding time dependent leakage constant (Ktrans) parametric map, Ktrans mean is 18.5; $e$ Axial T1-post contrast MRI brain 2 months after starting bevacizumab. $\mathbf{b}$ (bottom) Example of perfusion imaging in pseudoprogression. From left to right: a Axial T1-weighted post-contrast imaging from the initial post-operative MRI of the brain; $b$ Axial T1-weighted post-contrast image from the first MRI brain after completion of concurrent radiation and TMZ; $c$ Corresponding Vp parametric map, Vp mean is 2D. Corresponding Ktrans parametric map, Ktrans mean is 6.0; $e$ Axial T1weighted post-contrast image 6 months after completion of radiation therapy shows improvement in the enhancing part of the tumor consistent with pseudoprogression 


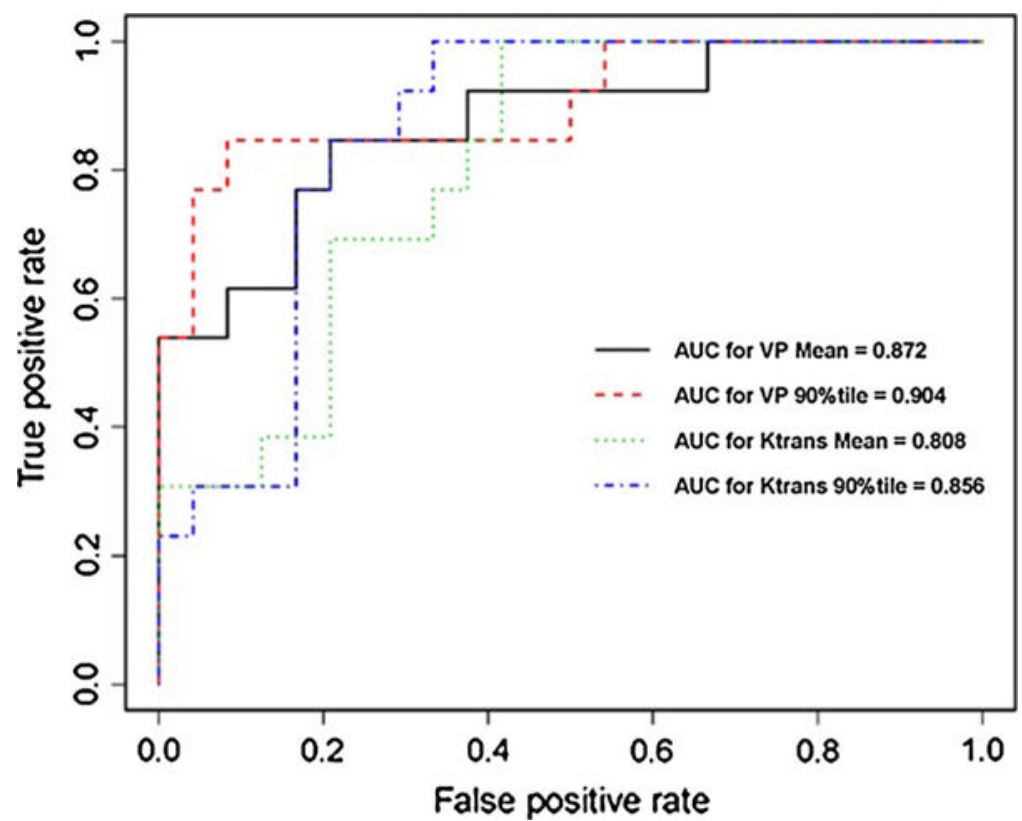

Fig. 3.

AUC curve for blood plasma volume (Vp) and Ktrans mean histogram analysis 


\section{Table 1}

Patient demographics

\begin{tabular}{llll}
\hline & Total & PD & PsP \\
\hline Patients, n (\%) & 37 & $24(65 \%)$ & $13(35 \%)$ \\
Median age (range) & $63(37-87)$ & $62(37-79)$ & $66(51-87)$ \\
Female gender, n (\%) & $12(32.4 \%)$ & $10(41.7 \%)$ & $2(15.4 \%)$ \\
Median KPS (range) & $90(60-100)$ & $80(60-100)$ & $90(75-100)$ \\
MGMT hypermethylation, n (\%) & $15($ of 36, 41.7\%) & $7(29.2 \%)$ & $8($ of $12,66.7 \%)$ \\
Median overall survival in months, $(95 \%$ CI) & $23.0(12.4-$ not reached) & $12.4(9.5-23.5)$ & $29.9(23.0-$ not reached) \\
\hline
\end{tabular}


Table 2

Analysis of relationship between perfusion parameter and progression status using Wilcoxon Rank-sum test

\begin{tabular}{lllc}
\hline \multirow{2}{*}{ Perfusion parameter } & \multicolumn{2}{l}{ Progression status (median, range) } & p value \\
\cline { 2 - 3 } & $\mathbf{P D}(\mathbf{n}=\mathbf{2 4})$ & $\mathbf{P s P}(\mathbf{n}=\mathbf{1 3})$ & \\
\hline $\mathrm{VP}_{\text {mean }}$ & $5.3(2.8-13.9)$ & $2.4(1.1-6.7)$ & 0.0002 \\
$\mathrm{VP}_{90} \%$ & $6.6(3.4-16.7)$ & $3.2(1.1-6.9)$ & $<0.0001$ \\
$\mathrm{Ktrans}_{\text {mean }}$ & $7.4(2.5-18.5)$ & $3.5(1.4-6.0)$ & 0.002 \\
Ktrans $_{90}$ & $9.1(2.9-22.2)$ & $4.2(1.4-6.5)$ & 0.0004 \\
\hline
\end{tabular}




\section{Table 3}

Analysis of relationship between changes (\%) of perfusion parameter (from baseline to follow-up) and progression status using Wilcoxon Rank-sum test

\begin{tabular}{|c|c|c|c|}
\hline \multirow[t]{2}{*}{ Perfusion parameter } & \multicolumn{2}{|c|}{ Median percent change (range) } & \multirow[t]{2}{*}{ p value } \\
\hline & PD $(n=24)$ & $\operatorname{PsP}(n=13)$ & \\
\hline $\mathrm{VP}_{\text {mean }}$ & $-8.2 \%(-74.4-164.8 \%)$ & $-32.5 \%(-71.3-172.0 \%)$ & 0.25 \\
\hline $\mathrm{VP}_{90 \%}$ & $-2.6 \%(-59.9-175.2 \%)$ & $-39.6 \%(-70.3-167.1 \%)$ & 0.02 \\
\hline Ktrans $_{\text {mean }}$ & $14.7 \%(-82.7-636.3 \%)$ & $18.6 \%(-58.7-225.7 \%)$ & 0.56 \\
\hline Ktrans $_{90} \%$ & $34.9 \%(-78.7-647.0 \%)$ & $3.8 \%(-56.2-199.9 \%)$ & 0.19 \\
\hline
\end{tabular}

